\title{
Remark on Immirzi Parameter, Torsion, and Discrete Symmetries
}

\author{
I.B. Khriplovich ${ }^{1}$ and A.A. Pomeransky ${ }^{2}$ \\ Budker Institute of Nuclear Physics \\ 630090 Novosibirsk, Russia, \\ and Novosibirsk University
}

\begin{abstract}
We point out that the new interaction of spinning particles with the torsion tensor, discussed recently, is odd under charge conjugation and time reversal. This explains rather unexpected symmetry properties of the induced effective 4-fermion interaction.
\end{abstract}

As has been demonstrated in [1, the gravitational action can be written as

$$
S_{g}=-\frac{1}{16 \pi G} \int d^{4} x e e_{I}^{\mu} e_{J}^{\nu}\left(R_{\mu \nu}^{I J}-\frac{1}{\gamma}{ }^{*} R_{\mu \nu}^{I J}\right)
$$

Here $I, J=0,1,2,3$ are internal Lorentz indices, $\mu, \nu=0,1,2,3$ are space-time indices; $e_{\mu}^{I}$ is the tetrad field, $e$ is its determinant, and $e_{I}^{\mu}$ is the object inverse to $e_{\mu}^{I}$; the curvature tensor is

$$
R_{\mu \nu}^{I J}=-\partial_{\mu} \omega_{\nu}^{I J}+\partial_{\nu} \omega_{\mu}^{I J}+\omega_{\mu}^{I K} \omega_{K}^{J}{ }_{\nu}-\omega_{\nu}^{I K} \omega_{K \mu}^{J},
$$

with its dual one

$$
{ }^{*} R_{\mu \nu}^{I J}=\frac{1}{2} \varepsilon_{K L}^{I J} R_{\mu \nu}^{K L}
$$

$\omega_{\mu}^{I J}$ is the connection. As to the so-called Immirzi parameter $\gamma$, the common belief is that it does not enter equations of motion since it appears as a factor at the term that vanishes on mass shell.

However, it was pointed out recently in [2] (see also earlier paper [3]) that in the presence of spin $1 / 2$ particles the second term in action (1) does not vanish on mass shell, and therefore $\gamma$ should enter the corresponding equations of motion.

Then it was pointed out in [4] (see also [5]) that the fermion action can be generalized as follows:

$$
S_{f}=\int d^{4} x e \frac{1}{2}\left[(1-i \alpha) \bar{\psi} \gamma^{I} e_{I}^{\mu} i \nabla_{\mu} \psi-(1+i \alpha) i \overline{\nabla_{\mu} \psi} \gamma^{I} e_{I}^{\mu} \psi\right]
$$

Here $\gamma^{I}$ are the Dirac matrices;

$$
\nabla_{\mu}=\partial_{\mu}-\frac{1}{4} \omega^{I J}{ }_{\mu} \gamma_{I} \gamma_{J}, \quad\left[\nabla_{\mu}, \nabla_{\nu}\right]=\frac{1}{4} R_{\mu \nu}^{I J} \gamma_{I} \gamma_{J}
$$

\footnotetext{
${ }^{1}$ khriplovich@inp.nsk.su

2 a.a.pomeransky@inp.nsk.su
} 
As to the real constant $\alpha$, it is of no consequence, generating a total derivative only, if the theory is torsion free, i.e. if

$$
\nabla_{[\mu} e_{\nu]}^{I}=0
$$

It is pointed out in [4, 5], however, that $\alpha$ becomes operative in the presence of the so-called contorsion tensor $C^{I J}{ }_{\mu}$ contributing to $\omega^{I J}{ }_{\mu}$, so that instead of (13) we have

$$
\nabla_{[\mu} e_{\nu]}^{I}=-C_{[\mu}^{I J} e_{\nu] J}
$$

In this case the structure generated by $\alpha$ does not reduce to the total derivative:

$$
e\left(\bar{\psi} \gamma^{I} e_{I}^{\mu} \nabla_{\mu} \psi+\overline{\nabla_{\mu} \psi} \gamma^{I} e_{I}^{\mu} \psi\right)=\partial_{\mu}\left(e e_{I}^{\mu} V^{I}\right)-e e_{I}^{\mu} C_{J \mu}^{I} V^{J}
$$

where $V^{J}$ is the vector current, $V^{J}=\bar{\psi} \gamma^{J} \psi$. Thus, nonvanishing $\alpha$ generates the effective interaction of spinning particles

$$
S_{\alpha}=-\frac{\alpha}{2} \int d^{4} x e e_{I}^{\mu} C_{J \mu}^{I} V^{J}=-\frac{\alpha}{2} \int d^{4} x e e_{I}^{\mu} C_{J \mu}^{I} \bar{\psi} \gamma^{J} \psi,
$$

new as compared to the canonical one.

Finally, the effective 4-fermion interaction obtained in this way in [4] is

$$
S_{f f}=\frac{3}{2} \pi G \frac{\gamma^{2}}{\gamma^{2}+1} \int d^{4} x e\left(A^{2}+\frac{2 \alpha}{\gamma} V A-\alpha^{2} V^{2}\right),
$$

where $A$ is the fermion axial current, $A^{I}=\bar{\psi} \gamma^{5} \gamma^{I} \psi$. For $\alpha=0$ and $\gamma \rightarrow \infty$, the result (77) goes over into that derived in [6, 7].

In connection with equation (17), the following question arises. The usual curvature tensor $R_{\mu \nu}^{I J}$ and its dual ${ }^{*} R_{\mu \nu}^{I J}$ behave in opposite ways both under the space reflection $P$ and the time reversal $T$ (see, e.g., [3]). To see it, we recall firstly that the duality transformation in electrodynamics results in $\mathbf{E} \rightarrow \mathbf{B}, \mathbf{B} \rightarrow-\mathbf{E}$, and that $\mathbf{E}$ and $\mathbf{B}$ behave in opposite ways both under $P$ and $T$. Then our duality problem in gravity is effectively reduced to that in electrodynamics by using the Petrov decomposition for the curvature tensor. Now, how it comes that the $T$-odd (and therefore $C P$-odd, in virtue of the $C P T$ theorem), $P$-odd second term in (1) generates the $P$-odd, but $T$-even $V A$ interaction in (17)?

The answer consists in rather special properties of the new effective interaction (6) introduced in [4, 5]. The factor $e_{I}^{\mu} C_{J \mu}^{I}$ therein is the same for fermions and antifermions, i.e. it is even under charge conjugation $C$. Then, the vector current $V^{J}=\bar{\psi} \gamma^{J} \psi$ is well-known to be $C$-odd. Thus, action (6) as a whole is $C$-odd too.

On the other hand, interaction (6) is also $T$-odd. Indeed, to simplify the arguments, let us consider a specific case when the covariant vector current $V_{J}$ has only a single, time component $\rho$, and $e_{I}^{\mu}$ reduces to $\delta_{I}^{\mu}$. Then

$$
e_{I}^{\mu} C_{J \mu}^{I} V^{J} \rightarrow C_{m}^{m t} \rho, \quad m=1,2,3 .
$$

Now, it can be easily demonstrated that the invariance of the scalar curvature $R$ under time reversal dictates that under this procedure $\omega_{m}^{m t}$ should change sign. Obviously, $C_{m}^{m t}$ has the 
same property. Since the particle density $\rho$ does not change under time reversal, interaction (6) is $T$-odd indeed.

Therefore, it is only natural that the $P$-odd and $T$-odd part of gravitational action (1), being combined with $T$-odd action (6), generates the $V A$ term in (77).

Our last remark refers to two other terms in the 4-fermion interaction (17). The coefficients at both are even functions of $1 / \gamma$, which means that they effectively arise via even powers of $P$ - and $T$-odd term in (1). Besides, the coefficient at $V^{2}$ is proportional to $\alpha^{2}$, i.e. is of second order in the $C$-odd interaction (6). No wonder therefore that both these terms are invariant under all the three discrete symmetries, $C, P$, and $T$. On the other hand, it is only natural in this respect that the coefficient at $V A$ is an odd function of $1 / \gamma$ and is linear in $\alpha$.

$* * *$

The investigation was supported in part by the Russian Foundation for Basic Research through Grant No. 05-02-16627.

\section{References}

[1] S. Holst, Phys. Rev. D53, 5966 (1996); gr-qc/9511026.

[2] A. Perez and C. Rovelli, gr-qc/0505081.

[3] R. Hojman, C. Mukku, and W.A. Sayed, Phys. Rev. D22, 1915 (1980).

[4] L. Freidel, D. Minic, and T. Takeuchi, hep-th/0507253.

[5] I.L. Shapiro, Phys. Rep. 357, 113 (2001); hep-th/0103093.

[6] H. Weyl, Phys. Rev. 77, 699 (1950).

[7] T.W.B. Kibble, J. Math. Phys. 2, 212 (1961). 